\title{
Estado nutricional e prevalência de anemia em crianças que freqüentam creches em Belo Horizonte, Minas Gerais
}

\author{
Nutritional status and anemia prevalence in children enrolled at day care centers in Belo Horizonte, \\ Minas Gerais, Brazil
}

Daniela da Silva Rocha ${ }^{1}$, Joel Alves Lamounier ${ }^{2}$,Flávio Diniz Capanema ${ }^{3}$, Sylvia do Carmo C. Franceschinit, Rocksane de Carvalho Norton ${ }^{5}$, Aline Bárbara P. Costa ${ }^{6}$, Maria Tereza G. Rodrigues ${ }^{6}$, Mariana Rodrigues de Carvalho ${ }^{6}$, Thaís Souza Chaves ${ }^{6}$

\section{RESUMO}

Objetivo: Avaliar o estado nutricional e a prevalência de anemia em crianças de sete a 74 meses, que freqüentam creches.

Métodos: Estudo transversal em 25 creches da regional leste de Belo Horizonte, Minas Gerais. Realizaram-se avaliação antropométrica e dosagem de hemoglobina por hemoglobinômetro portátil. Considerou-se anemia se hemoglobina $<11,0 \mathrm{~g} / \mathrm{dL}$ para crianças menores que 60 meses e $<11,5 \mathrm{~g} / \mathrm{dL}$, para maiores de 5 anos. $\mathrm{Na}$ avaliação do estado nutricional, utilizaram-se os índices peso/ idade, estatura/idade e peso/estatura, sendo categorizados em três intervalos: abaixo de -2 escores $Z$ (desnutrição/baixa estatura), de -2 a - 1 escore $Z$ (risco para desnutrição/baixa estatura) e maior ou igual a -1 escore Z. A análise estatística incluiu: qui-quadrado, qui-quadrado de tendência linear e análise de variância (ANOVA) seguida pelo teste de Tukey, sendo significante $p<0,05$.

Resultados: Foram avaliadas 402 crianças, com mediana de idade de 46,3 meses. A prevalência de anemia foi 28,8\%, observando-se o dobro da prevalência nas crianças com idade inferior a 24 meses (70,4\%), comparada às de faixa etária maior. A prevalência de desnutrição correspondeu a 5,0 e 5,5\% da população para os índices peso/estatura e peso/ idade, respectivamente. A prevalência de baixa estatura foi $4,2 \%$. Em crianças menores de 5 anos, a anemia associou-se à idade e ao deficit estatural.

Conclusões: A anemia em crianças matriculadas nas creches da regional leste de Belo Horizonte constitui importante problema de saúde pública, acometendo, principalmente, os menores de 24 meses. Os deficits nutricionais estiveram acima dos valores esperados.

Palavras-chave: anemia; estado nutricional; creches; criança; Saúde Pública.

\section{ABSTRACT}

Objective: Assess the prevalence of anemia and the nutritional status of children with 7-74 months old, enrolled in day care centers.

Methods: This cross-sectional study enrolled children of 25 day care centers of the eastern district of Belo Horizonte city, Minas Gerais, Brazil. Anthropometric parameters were evaluated and hemoglobin count was done by a portable hemoglobinometer. The cutoff level for anemia was $11.0 \mathrm{~g} / \mathrm{dL}$ for children less than 60 months, and $11.5 \mathrm{~g} / \mathrm{dL}$ for those older than 5 years old. Nutritional status was assessed by anthropometric indexes: weight-for-age, height-for-age and weight-for-height and classified in 3 groups: below -2 Z score (malnutrition/height deficit), -2 to $-1 \mathrm{Z}$ score (nutritional risk for malnutrition/height deficit) and above or equal to $-1 \mathrm{Z}$ score. Statistical analysis included: chi-square, chi-square with linear trend, analysis of variance (ANOVA) followed by Tukey test, being significant $p<0.05$.

Results: 402 children, with a median age of 46.3 months, were assessed. The overall anemia prevalence was $28.8 \%$. The
${ }^{1}$ Mestre em Ciências da Saúde pela Faculdade de Medicina da Universidade Federal de Minas Gerais (UFMG) e professora do curso de Nutrição do Centro Universitário UNA e do Centro Universitário Metodista Izabela Hendrix, Belo Horizonte, MG, Brasil

${ }^{2}$ Doutor em Saúde Pública e Nutrição pela Universidade da Califórnia, Los Angeles e professor titular do Departamento de Pediatria da Faculdade de Medicina da UFMG, Belo Horizonte, MG, Brasil

${ }^{3}$ Doutor em Ciências da Saúde pela Faculdade de Medicina da UFMG, professor da Faculdade da Saúde e Ecologia Humana, Vespasiano, MG, Brasil ${ }^{4}$ Doutora em Nutrição pela Universidade Federal de São Paulo e chefe da Divisão de Saúde da Universidade Federal de Viçosa, MG, Brasil

${ }^{5}$ Doutora em Medicina e professora adjunta do Departamento de Pediatria da Faculdade de Medicina da UFMG, Belo Horizonte, MG, Brasil

${ }^{6}$ Graduanda em Nutrição pela UFMG, Belo Horizonte, MG, Brasil
Endereço para correspondência:

Terezinha Soares Biscegli

Rua Fortaleza, 95 - Jardim Ferreira

CEP 15840-000 - Itajobi/SP

E-mail: terezinhabiscegli@yahoo.com.br

Fonte financiadora: Fundação de Amparo à Pesquisa do Estado de Minas Gerais (Fapemig) e Furnas Centrais Elétricas

Recebido em: 25/7/2007

Aprovado em: 18/9/2007 
rate of anemia in children $<24$ months $(70.4 \%)$ was twice that observed in older children. The prevalence of malnutrition was 5,0 and $5.5 \%$, as defined by weight-for-height and weight-for-age indexes, respectively. The prevalence of height deficit was $4.2 \%$. Malnutrition was associated to age and height deficit in children $<5$ years old.

Conclusions: Anemia in children enrolled in day care centers in Belo Horizonte is a serious public health problem, especially in those younger than 24 months. Nutritional deficits were beyond the expected values.

Key-words: anemia; nutritional status; child day care centers; child; Public Health.

\section{Introdução}

Em países em desenvolvimento, a desnutrição energéticoprotéica e a anemia ferropriva em crianças são carências nutricionais de grande importância e merecem especial atenção por parte dos profissionais que atuam na área da saúde.

As condições nutricionais da população infantil no país apontam para uma evolução favorável entre 1974 e 1996, com redução da prevalência de deficit de crescimento, porém com diferenças significativas entre as regiões brasileiras. $\mathrm{O}$ declínio da desnutrição pode ser explicado pela melhoria das condições socioeconômicas, escolaridade materna, saneamento básico e pelo maior acesso a serviços de saúde ${ }^{(1)}$.

Apesar da anemia, ao contrário da desnutrição, estar presente em todas as classes sociais, seu risco é maior em famílias de baixa renda ${ }^{(2)}$, configurando-se como uma endemia, sob a ótica da Saúde Pública, já que, segundo estimativas, acomete mais de 3 bilhões de pessoas ${ }^{(3)}$. Este problema é ainda maior ao se considerar a deficiência de ferro, pois, para cada caso de anemia ferropriva diagnosticado, estima-se o dobro de casos de deficiência de ferro ocultos na população ${ }^{(4)}$.

Diversos estudos demonstram que a anemia é também um grave problema de Saúde Pública no Brasil, com prevalência elevada, variando de 46,3 a 73,2\%(5,6). Em estudo conduzido por Spinelli et a ${ }^{(6)}$ em 12 centros urbanos das cinco regiões geográficas do país, encontrou-se prevalência média de 65,4\%. A maior prevalência de anemia foi encontrada no Sudeste $(70,4 \%)$, apesar de ser a região de maior concentração de riqueza do país. Já a menor prevalência foi detectada na região Sul (59,7\%).

As consequiências da anemia na infância variam de fadiga, perda de apetite e maior suscetibilidade às infecções até atraso no desenvolvimento cognitivo e motor ${ }^{(7)}$, que pode perdurar mesmo após o tratamento medicamentoso ${ }^{(8)}$. Várias medidas de caráter populacional têm sido apresentadas com o intuito de reduzir a prevalência de anemia em nosso meio, como a fortificação de alimentos ${ }^{(9,10)}$, a suplementação de ferro para crianças com menos de dois anos, consideradas as de maior risco de anemia $^{(11)}$ e o uso de doses intermitentes de ferro para reduzir os efeitos adversos e propiciar maior adesão ao tratamento ${ }^{(12)}$. Além dessas medidas, o Ministério da Saúde determinou a obrigatoriedade da adição de ferro e ácido fólico às farinhas de milho e trigo, como uma estratégia de alcance populacional ${ }^{(13)}$. Porém, a efetividade dessas ações é ainda desconhecida e os resultados obtidos não parecem corresponder às expectativas.

A relação entre a anemia e deficit pôndero-estatural na infância não foi definitivamente estabelecida, apesar de a anemia poder interferir negativamente no apetite de crianças. Alguns estudos mostram tal associação ${ }^{(2,14)}$. Brunken et al ${ }^{(14)}$, avaliando 271 crianças com menos de 36 meses de idade assistidas em creches públicas de Cuiabá, Mato Grosso, observaram redução proporcional dos índices antropométricos e da concentração de hemoglobina. Em crianças com deficit de peso para idade, a prevalência de anemia foi 1,73 vezes maior (IC95\% $=1,55-1,93$ ) e, naquelas com deficit de estatura, a anemia foi 1,27 vezes mais freqüente (IC95\%=1,06-1,53), comparadas àquelas sem deficits. Os autores sugeriram que crianças com quadro de desnutrição crônica são mais suscetíveis à deficiência de ferro. Entretanto, Almeida et a ${ }^{(15)}$ não encontraram associação entre anemia e estado nutricional em 192 crianças atendidas em creches de Pontal, São Paulo, mas observaram elevada prevalência de anemia em uma população com baixa prevalência de desnutrição.

Os estudos sobre a influência das creches na saúde das crianças são inconclusivos ${ }^{(16-19)}$. Algumas pesquisas mostraram que crianças que freqüentam creches apresentam maior risco de desenvolver diarréia e doenças infecciosas respiratórias ${ }^{(18,19)}$. Por outro lado, várias investigações ressaltam a importância das creches na melhoria do estado nutricional das crianças, principalmente para aquelas de menor renda familiar ${ }^{(2,16,20)}$. Os benefícios da assistência a pré-escolares nas creches estão relacionados à melhor oferta nutricional, aos processos de socialização e estímulo psicomotor, além do apoio à família para a guarda segura de seus filhos ${ }^{(21)}$.

Assim, torna-se importante conhecer o estado nutricional e a prevalência de anemia em crianças que freqüentam creches públicas. O objetivo do presente estudo foi analisar a prevalência de anemia e de deficit nutricional em crianças assistidas nas creches da regional leste da cidade de Belo Horizonte, Minas Gerais, Brasil. 


\section{Métodos}

Estudo de corte transversal, realizado nos meses de junho e julho de 2005 , com crianças de sete a 74 meses de idade, matriculadas em período integral nas creches pertencentes ou conveniadas à Prefeitura Municipal de Belo Horizonte, localizadas na área de abrangência administrativa da regional leste do município. Em cada creche foi agendada uma reunião com os pais e/ou responsáveis para informar sobre a pesquisa e distribuir folheto explicativo sobre anemia e suas conseqüências.

O projeto foi aprovado pela Câmara Departamental do Departamento de Pediatria da Universidade Federal de Minas Gerais (UFMG), pelo Comitê de Ética em Pesquisa da UFMG e pelo Comitê de Ética em Pesquisa da Secretaria Municipal de Saúde de Belo Horizonte. Os pais e/ou responsáveis assinaram o termo de consentimento para a participação das crianças na pesquisa.

A regional leste conta com 2.646 crianças distribuídas em duas creches próprias pertencentes à prefeitura e outras 24 conveniadas, abrangendo um total de 13,2\% das crianças atendidas em creches públicas do município de Belo Horizonte. Do total de creches contatadas, uma não foi incluída no estudo, pois funcionava em período parcial. A população residente na regional leste apresenta características heterogêneas em relação à condição socioeconômica, sendo constituída tanto por indivíduos em condições precárias de vida como por outros provenientes de famílias com bom poder aquisitivo. Porém, as crianças atendidas nas referidas creches pertencem às classes socioeconômicas de menor poder aquisitivo.

A amostra foi calculada com base no número de crianças com mais de seis meses, regularmente inscritas em período integral nas 25 creches da regional leste de Belo Horizonte $(\mathrm{n}=2.344$ crianças). Para o cálculo da amostra, utilizou-se a prevalência de anemia estimada de $40 \%$, com precisão de $5 \%$ e intervalo de confiança de 95\%, resultando em 319 crianças. A esse valor, acrescentou-se um mínimo de $20 \%$ para compensar possíveis perdas ( $\mathrm{n}=382$ crianças). Tal cálculo foi feito com o programa Statcal do software Epi-Info 6.04.

As crianças foram selecionadas por randomização, com o uso do programa Excel 2003. O número de crianças sorteadas em cada creche foi estabelecido pelo peso que cada creche representava em relação ao total de crianças matriculadas nas 25 creches $(\mathrm{n}=2.344)$. O tamanho da amostra foi de 407 crianças. Desse total, cinco foram excluídas por apresentarem dados incompletos. Todas as crianças sortea- das e que estavam presentes no dia do exame, sem sinais e sintomas de doenças infecciosas como febre, tosse, diarréia e/ou vômitos, foram incluídas no estudo.

Os dados de identificação (nome e data de nascimento) foram coletados dos prontuários das creches. As características familiares e socioeconômicas foram obtidas por meio de questionários padronizados aplicados em entrevistas aos pais ou responsáveis. As entrevistas foram realizadas nas creches, na entrada ou saída das crianças.

Os dados antropométricos de peso e estatura foram coletados por estagiárias do curso de nutrição, de acordo com as técnicas estabelecidas pela Organização Mundial da Saúde (OMS) ${ }^{(22)}$, sendo supervisionadas pela nutricionista responsável pela pesquisa. Para obter o peso corporal, as crianças se apresentavam com o mínimo de roupas. O peso foi verificado em balança digital eletrônica, com capacidade para $150 \mathrm{~kg}$ e divisão de $100 \mathrm{~g}$. O comprimento das crianças com menos de 24 meses foi medido em infantômetro (modelo AlturExata $\left.{ }^{\circledR}\right)$. As crianças com idade superior a 24 meses foram medidas em pé, descalças, após adaptação do infantômetro para estadiômetro.

O estado nutricional foi avaliado por meio dos índices peso/idade, estatura/idade e peso/estatura, expressos em escore Z. Os índices antropométricos foram categorizados em três intervalos: <-2 escore $Z$, desnutrição; $-2 \geq$ escore $Z<-1$, risco nutricional; $\geq-1$ escore $Z$, eutrofia. Como referências, foram adotadas as curvas de crescimento do Centers for Disease Control and Prevention (CDC $)^{(23)}$. Para a análise dos dados antropométricos, foi utilizado o software Epi-Info, versão 2002.

Para avaliar a anemia, uma amostra de sangue de cada criança foi coletada em microcuvetas descartáveis por punção capilar. A concentração de hemoglobina foi medida com um fotômetro portátil (hemoglobinômetro) da marca HemoCue ${ }^{\circledR}$. Foram consideradas anêmicas as crianças com hemoglobina inferior a $11,0 \mathrm{~g} / \mathrm{dL}$ para faixa etária entre seis e 59 meses. Naquelas maiores de 60 meses, o valor estabelecido foi $<11,5 \mathrm{~g} / \mathrm{dL}^{(24)}$. De acordo com de Maeyer ${ }^{(25)}$, foram utilizadas as seguintes categorias para definir os graus de anemia: anemia grave se hemoglobina $<7,0 \mathrm{~g} / \mathrm{dL}$; anemia moderada se hemoglobina entre 7,0 e $9,0 \mathrm{~g} / \mathrm{dL}$; e anemia leve se hemoglobina $>9,0 \mathrm{~g} / \mathrm{dL}$ e $<11,0 \mathrm{~g} / \mathrm{dL}$.

Para compilação e análise dos dados, utilizou-se o programa Epi-Info, versão 6.04b. Para a análise estatística foram empregados os seguintes testes: qui-quadrado para variáveis dicotômicas e qui-quadrado de tendência linear para três ou mais grupos, além da Odds Ratio (OR) para avaliar a magnitude 
das associações. Realizou-se o teste de normalidade (Kolmogorov-Smirnov) para as médias de hemoglobina em todas as faixas etárias, as quais apresentaram distribuição normal. As médias foram comparadas pelo teste $t$ no caso de dois grupos independentes. Aplicou-se a Análise de Variância (ANOVA) complementada com o procedimento de comparações múltiplas de Tukey, para três ou mais grupos independentes. O nível de rejeição da hipótese de nulidade foi inferior a 0,05.

\section{Resultados}

Foram avaliadas 402 crianças com mediana de idade de 46,2 meses, variando de 7,3 a 73,5 meses. A freqüência de crianças do sexo masculino foi $51,7 \%$. A média de idade das mães foi $29 \pm 6$ anos e $43,3 \%$ delas tinham o primeiro grau incompleto. Em relação à renda per capita, 64,6\% das famílias relataram receber valor igual ou inferior a $\mathrm{R} \$ 150,00$, com mediana de $\mathrm{R} \$ 133,00$. A maior parte das crianças morava com o pai e a mãe $(53,8 \%)$, seguidas daquelas que moravam com a mãe $(41,9 \%)$ e o restante morava com os avós ou outros parentes $(4,3 \%)$.

A prevalência de anemia nas 402 crianças e a média de hemoglobina por faixa etária está exposta na Tabela 1. Anemia na forma grave ocorreu em uma criança, na forma moderada em seis crianças e a forma leve foi observada em $27,1 \%$ das crianças estudadas. Das crianças com anemia moderada, cinco tinham idade inferior a 24 meses e uma entre 24 e 48 meses. A média de hemoglobina no grupo de crianças com menos de 24 meses foi significantemente menor, comparada às demais faixas etárias. As crianças de 24-48 meses também apresentaram nível médio de hemoglobina significantemente menor do que aquelas com mais de 60 meses $(p<0,013)$.

A prevalência de desnutrição e risco nutricional para o índice peso/estatura foi de 5,0 e 12,9\%; respectivamente. Para o índice peso/idade, os valores foram de 5,5 e $15,7 \%$ para desnutrição e risco nutricional, respectivamente. A prevalência de baixa estatura foi de $4,2 \%$ e de risco nutricional para baixa estatura foi de $16,4 \%$.

A relação entre as médias dos índices antropométricos e a prevalência de anemia por idade é apresentada na Tabela 2. Esta análise levou em conta dois grupos de idade $(<60$ meses versus $\geq 60$ meses), devido ao ponto de corte para anemia ser diferente em ambos os grupos. Observou-se que as crianças anêmicas apresentaram médias de escore $\mathrm{Z}$ para peso/altura inferiores às crianças não-anêmicas, com exceção das menores de 60 meses, porém sem significância

Tabela 1 - Prevalência de anemia e hemoglobina média, segundo a faixa etária

\begin{tabular}{ccccccc}
\hline $\begin{array}{c}\text { Faixa etária } \\
\text { (meses) }\end{array}$ & \multicolumn{2}{c}{ Anêmicos } & \multicolumn{2}{c}{ Não anêmicos } & Hemoglobina (g/dL) & ANOVA \\
\hline$\leq 24$ & $\mathbf{N}$ & $\%$ & $\mathbf{N}$ & $\%$ & $10,4 \pm 1,4$ & $<0,001^{*}$ \\
$24-\mid 48$ & 30 & 71,4 & 12 & 28,6 & $11,8 \pm 1,3$ & $0,013^{* *}$ \\
$48-60$ & 46 & 26,1 & 130 & 73,9 & $12,1 \pm 1,2$ & \\
$\geq 60$ & 20 & 21,3 & 74 & 78,7 & $12,3 \pm 1,2$ & \\
\hline
\end{tabular}

${ }^{*} \leq 24$ menor do que 24-|48, 48-60 e $\geq 60$ meses; ${ }^{*} 24-\mid 48$ menor que do que $\geq 60$ meses

Tabela 2 - Análise da associação entre os índices antropométricos de crianças anêmicas e não anêmicas, segundo a faixa etária

\begin{tabular}{lcccc}
\hline $\begin{array}{l}\text { Indicadores } \\
\text { antropométricos }\end{array}$ & $\begin{array}{c}\text { Faixa etária } \\
\text { (meses) }\end{array}$ & Anêmicos & Não anêmicos & Teste $\boldsymbol{t}$ \\
\hline Peso/idade & $<60$ & $-0,31 \pm 1,08$ & $-0,13 \pm 1,13$ & 0,18 \\
& $\geq 60$ & $-0,72 \pm 1,15$ & $-0,16 \pm 0,99$ & 0,08 \\
Altura/idade & $<60$ & $-0,36 \pm 0,92$ & $-0,07 \pm 1,07$ & 0,02 \\
& $\geq 60$ & $-0,36 \pm 0,87$ & $-0,06 \pm 1,06$ & 0,36 \\
Peso/altura & $<60$ & $0,03 \pm 1,12$ & $-0,08 \pm 1,05$ & 0,43 \\
& $\geq 60$ & $-0,79 \pm 1,59$ & $-0,11 \pm 0,99$ & 0,16 \\
\hline
\end{tabular}


estatística. A média do indicador altura/idade, nas crianças menores de 60 meses, foi significantemente menor nas anêmicas em relação às não-anêmicas $(p=0,02)$.

A Tabela 3 apresenta a razão de chances de anemia segundo algumas variáveis biológicas estudadas. Idade inferior a 24 meses e relação estatura/idade entre -2 Z-escore e -1 Z-escore se associaram à anemia nas crianças.

\section{Discussão}

A prevalência de anemia encontrada no presente estudo mostra que tal carência nutricional ainda é um problema de Saúde Pública a ser considerado em creches de Belo Horizonte. Apesar disso, essa cifra foi inferior à relatada por alguns autores. Brunken $e a^{\left(l^{14)}\right.}$, ao avaliarem crianças menores de 36 meses atendidas em creches públicas de Cuiabá, encontraram prevalência de $63 \%$ de anemia. Almeida et al ${ }^{(15)}$, estudando 192 crianças entre 12 e 72 meses atendidas em creches da cidade de Pontal, encontraram prevalência de 62,5\% de anemia. Nas cidades de São Paulo e Rio de Janeiro foram observadas prevalências de anemia de $68,8^{(26)}$ e $47,3 \%{ }^{(27)}$, respectivamente. É possível que a menor prevalência de anemia em crianças de creches de Belo Horizonte possa ser atribuída à menor freqüência de crianças com menos de 24 meses na presente investigação, comparada às demais. Entretanto, são necessários mais estudos para avaliar possíveis fatores protetores, tais como tipo de alimentação oferecida e de assistência prestada às crianças nas creches de Belo Horizonte.

Ao avaliar a prevalência de anemia por faixa etária, observou-se que esta foi mais que o dobro nas crianças com menos de 24 meses $(71,4 \%)$, comparadas às outras faixas de idade. O valor médio de hemoglobina encontrado nos menores de 24 meses ficou abaixo do ponto de corte para anemia $(<11,0 \mathrm{~g} / \mathrm{dL})$, ao contrário das outras faixas etárias. Há um consenso na literatura de que a idade é um forte fator de risco para anemia e os estudos que avaliaram anemia por faixa etária mostraram maior prevalência de anemia em

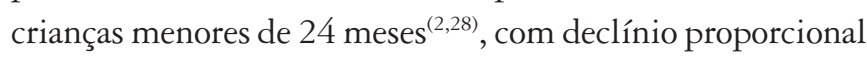
à maior idade. Tal achado se associa ao ritmo de crescimento acelerado nos dois primeiros anos de vida, levando à maior necessidade de ferro, aliada a uma dieta monótona, excessiva em cálcio e insuficiente em ferro e vitamina $C^{(29)}$. No pre-

Tabela 3 - Razão de chances e intervalo de confiança de 95\% (OR e IC95\%) para a presença de anemia nas crianças $<60$ meses de idade, segundo características demográficas e nutricionais

\begin{tabular}{lccc}
\hline Características & Número & OR & IC95\% \\
\hline $\begin{array}{l}\text { Sexo } \\
\text { Feminino }\end{array}$ & 150 & 1,00 & \\
$\quad$ Masculino & 162 & 1,05 & $0,63-1,75$ \\
Faixa etária* & & & \\
$48-\mid 59$ & 94 & 1,00 & $0,69-2,49$ \\
$24-\mid 48$ & 176 & 1,31 & $3,74-23,31$ \\
$\leq 24$ meses & 42 & 9,25 & \\
Altura/idade* & & & $1,09-3,97$ \\
$\geq-1$ & 243 & 1 & $0,32-5,02$ \\
$-2 \leq Z<-1$ & 57 & 2,05 & \\
$<-2$ & 12 & 1,31 & \\
Peso/idade* & & & $0,59-2,34$ \\
$\geq-1$ & 245 & 1 & $0,44-4,43$ \\
$-2 \leq Z<-1$ & 51 & 1,18 & \\
$<-2$ & 16 & 1,41 & $0,64-2,97$ \\
Peso/altura* & & & $0,37-4,49$ \\
$\geq-1$ & 254 & 1,39 & \\
$-2 \leq Z<-1$ & 38 & 1,32 & \\
$<-2$ & 14 & & \\
\hline
\end{tabular}

*Qui-quadrado de tendência linear 
sente estudo, as crianças com menos de dois anos e aquelas entre 24 e 48 meses apresentaram 9,25 e 1,31 vezes mais chance de anemia em relação àquelas entre 48 e 59 meses respectivamente, em consonância com os resultados obtidos por outros autores ${ }^{(28,30)}$.

A avaliação do estado nutricional revelou que o deficit de maior freqüência na população estudada foi o de peso/idade $(5,5 \%)$, seguido pelo peso/estatura $(5 \%)$ e estatura/idade $(4,2 \%)$. Os deficits apresentados pelas crianças encontram-se acima do esperado, considerando-se que, em uma população com boas condições de saúde e nutrição, aproximadamente $2,3 \%$ dos valores devem estar no primeiro intervalo, $13,6 \%$ no segundo intervalo e o restante, $84,1 \%$, devem apresentar escore $Z \geq-1^{(17)}$. Ressalta-se que, para crianças com mais 24 meses, os indicadores antropométricos mais adequados para a avaliação nutricional são o peso/estatura e a estatura/ idade, sendo o peso/idade apropriado para crianças menores de 24 meses, quando o crescimento ponderal é mais acentuado.

Os valores encontrados no presente estudo diferem dos demais disponíveis na literatura, nos quais o deficit estatural é o indicador antropométrico menos satisfatório ${ }^{(14,16,17)}$. Fisberg et $a^{(31)}$, ao avaliarem 827 crianças menores de 84 meses em São Paulo, concluíram que o deficit estatural foi o índice nutricional mais inadequado, com prevalência de $7 \%$, enquanto a prevalência de desnutrição foi de apenas $0,9 \%$ ao se empregar o índice peso/estatura. Porém, Netto ${ }^{(32)}$, ao avaliar crianças de 18 a 24 meses do município de Viçosa, Minas Gerais, encontrou prevalência de 9,9\% de desnutrição segundo o indicador peso/estatura, 7,9\% para peso/idade e $3 \%$ de deficit de estatura/idade.

Em relação ao risco de deficit nutricional, com exceção do índice peso/idade, os valores estão acima do esperado segundo a população de referência $(13,6 \%)^{(17)}$, sendo o mais insatisfatório o índice estatura/idade $(16,4 \%)$, seguido pelo peso/idade $(15,7 \%)$ e peso/estatura $(12,9 \%)$. Esses dados estão de acordo com os encontrados por Silva $e t$ a $l^{(17)}$ que, ao analisarem dados da Pesquisa Nacional de Saúde e Nutrição (PNSN, 1989) constituída por 10.667 crianças menores de seis anos, encontraram prevalência de risco nutricional de 24,6\% para estatura/idade, 23,4\% para peso/idade e $9,74 \%$ para peso/estatura. Silva e Sturion ${ }^{(16)}$ também avaliaram 2.096 crianças atendidas em centros educacionais e creches no município de Piracicaba, São Paulo, e encontraram maior risco nutricional para o índice estatura/idade, com prevalência de $17,4 \%$.

No presente estudo, a análise da relação entre estado nutricional e prevalência de anemia mostrou valores infe- riores nas médias de escore $\mathrm{Z}$ dos índices antropométricos nas crianças anêmicas em relação às não anêmicas, porém sem diferenças significantes - com exceção das menores de 60 meses para o índice peso/estatura. Nestas, o índice altura/idade apresentou diferença significante entre crianças anêmicas e não anêmicas, demonstrando relação entre desnutrição crônica e anemia. A relação entre deficit nutricional e anemia é ainda bastante controversa. Schmitz et al ${ }^{(2)}$ avaliaram 279 crianças menores de 36 meses de baixa renda, assistidas em creches de Brasília, Distrito Federal, verificando que, entre as crianças não anêmicas, a prevalência de desnutrição foi $3 \%$. Já entre as anêmicas, esse percentual se elevou para $11,2 \%$. Netto et al $^{(33)}$ avaliaram 206 crianças maiores de um ano no município de Viçosa e também encontraram maior prevalência de anemia nas crianças desnutridas, considerando todos os índices antropométricos. Matta et a ${ }^{(27)}$ observaram que as crianças anêmicas apresentam menores médias de escore $Z$ segundo os índices peso/idade e altura/idade, sendo essas diferenças estatisticamente significantes. Por outro lado, Neuman et al ${ }^{(34)}$, no sul do país, não encontraram relação entre essas duas carências nutricionais.

Uma das hipóteses que explica a relação entre anemia e desnutrição é o fato de as duas entidades apresentarem fatores de risco comuns, tais como inadequação da dieta, falta de saneamento básico, dificuldades de acesso à assistência à saúde e baixo nível de escolaridade dos pais. Entretanto, ao analisar o quadro epidemiológico dessas duas carências nutricionais em separado, observa-se que, ao contrário da desnutrição, as taxas de anemia vêm aumentando ao longo dos anos, constituindo-se em grave problema de saúde pública em várias regiões do país, apesar da melhora de alguns indicadores socioeconômicos ${ }^{(29)}$.

Não foi encontrada, nesse estudo, relação entre sexo e anemia, confirmando os achados de estudos semelhantes ${ }^{(28,35)}$. Já Oliveira et $a^{(36)}$, ao avaliarem 1.287 crianças pré-escolares da Paraíba, observaram que os pré-escolares do sexo masculino apresentaram prevalência de anemia significantemente maior $(40,8 \%$; IC $=36,9-44,8 \%)$ do que o feminino $(32,2 \%$; IC $=28,7-35,9 \%)$. Segundo Torres et al ${ }^{(37)}$, a maior prevalência de anemia no sexo masculino pode ser explicada pela maior velocidade de crescimento dos meninos em relação às meninas, acarretando maior necessidade de ferro pelo organismo.

Os dados obtidos no presente estudo revelam uma situação preocupante em relação à prevalência de anemia, principalmente na faixa etária inferior a 24 meses. 
Mas, mesmo em crianças maiores, a anemia deve ser considerada um problema de saúde pública, de acordo com a OMS. Em relação ao estado nutricional, observou-se que os deficits e o risco de deficits nutricionais estão acima do padrão de referência. Tais dados demonstram a necessidade de implantar e implementar medidas efetivas para prevenir e controlar essas carências nutricionais nas crianças assistidas em creches de Belo Horizonte. Uma medida importante, implementada após esse diagnóstico de situação nutricional nas creches de Belo Horizonte, foi fortificar a água com ferro e vitamina $C$; os resultados dessa ação serão publicados posteriormente. Outra medida importante, principalmente para crianças com menos de dois anos de idade, é o estímulo ao aleitamento materno, reforçando sua importância para o desenvolvimento e o crescimento das crianças.

\section{Agradecimentos}

Agradecemos às alunas Flaviane Alves Toni, Lívia Salomé de Oliveira, Verena Bartrowiak Oliveira, Patrícia de Vasconcelos Sadala, Suellen Fabiane Campos pela ajuda na coleta de dados. Ao professor Mark Anthony Beinner, agradecemos pela ajuda na tradução do Abstract. Agradecemos também ao Centro de Pós-Graduação da UFMG pelo apoio técnico e a todas as funcionárias das creches participantes do estudo pelo carinho demonstrado em relação à equipe de trabalho.

\section{Referências bibliográficas}

1. Monteiro CA, Benício MH, lunes RF, Gouveia NC, Cardoso MA. Evolução da desnutrição infantil. In: Monteiro CA, editor. Velhos e novos males da saúde no Brasil. São Paulo: Hucitec; 1995. p. 93-115.

2. Schmitz BAS, Picanço MR, Aquino KKNC, Bastos J, Giorgini E, Cardoso R et al. Prevalência de desnutrição e anemia em pré-escolares de Brasília (Brasil). Pediatr Moderna 1998;34:155-64.

3. World Health Organization Feeding and Nutrition of Infants and Young Children - Regional Publications, n 87. Copenhagen: World Health Organization; 2003.

4. Yip R. Iron deficiency: contemporary scientific issues and international programmatic approaches. J Nutr 1994;124 (Suppl 8):S1479-90.

5. Assis AM, Barreto ML, Gomes GS, Prado Mda S, Santos NS, Santos LM et al. Childhood anemia prevalence and associated factors in Salvador, Bahia, Brazil. Cad Saude Publica 2004;20:1633-41.

6. Spinelli MG, Marchioni DM, Souza JM, Souza SB, Szarfarc SC. Fatores de risco para anemia em crianças de 6 a 12 meses no Brasil. Rev Panam Salud Publica 2005;17:84-91.

7. Grantham-Mcgregor S, Ani C.A review of studies on the effect of iron deficiency on cognitive development in children. J Nutr 2001;131:649-68.

8. Lozoff B, Jimenez E, Hagen J, Mollen E, Wolf AW. Poorer behavioral and developmental outcome more than 10 years after treatment for iron deficiency in infancy. Pediatrics 2000;105:E51.

9. Tuma RB, Yuyama LKO, Aguiar JPL, Marques HO. Impacto da farinha de mandioca fortificada com ferro aminoácido quelato no nível de hemoglobina de pré-escolares. Rev Nutr 2003;16:29-39.

10. Beinner MA, Lamounier JA, Tomaz C. Effect of iron-fortified drinking water facilities on the hemoglobin status of young children. J Am Coll Nutr 2005;24:107-14.

11. Ribeiro LC, Devincenzi MU, Sigulem DM. Anemia ferropriva na primeira infância. Compacta Nutrição [serial on the Internet]. 2000;1(1):1-17 [cited 2007 Oct 8]. Available from: http://www.pnut.epm.br/Download_Files/nutricao.pdf.

12. Brunken GS, Muniz PT, Silva SM. Weekly iron supplementation reduces anemia prevalence by $1 / 3$ in preschool children. Rev Bras Epidemiol 2004;7:210-9.
13. ANVISA [homepage on the Internet]. Fabricantes de farinhas terão de adicionar ferro e ácido fólico ao produto da Resolução-RDC n³44, de 13 de dezembro de 2002 [cited 2007 Oct 08]. Available from: http://e-legis.anvisa.gov.br/leisref/public

14. Brunken GS, Guimarães LV, Fisberg M. Anemia em crianças menores de 3 anos que freqüentam creches públicas em período integral. J Pediatr (Rio J) 2002;78:50-6

15. Almeida CA, Ricco RG, Ciampo LA, Souza AM, Pinho AP, Oliveira JE. Fatores associados à anemia por deficiência de ferro em crianças pré-escolares brasileiras. J Pediatr (Rio J) 2004;80:229-34.

16. Silva MV, Sturion GL. Freqüência à creche e outros condicionantes do estado nutricional infantil. Rev Nutr 1998;11:58-68.

17. Silva MV, Ometto AMH, Furtuoso COM, Pipitone MAP, Sturion GL. Acesso à creche e estado nutricional das crianças brasileiras: diferenças regionais, por faixa etária e classes de renda. Rev Nutr 2000;13:193-9.

18. Barros AJ. Child-care attendance and common morbidity: evidence of association in the literature and questions of design. Rev Saude Pubica 1999;3:98-106.

19. Vico ES, Laurenti R. Mortalidade de crianças usuárias de creches no município de São Paulo. Rev Saude Publica 2004;38:38-44.

20. Bueno MB, Marchioni DM, Fisberg RM. Evolução nutricional de crianças atendidas em creches públicas no município de São Paulo, Brasil. Rev Panam Salud Publica 2003;14:165-70.

21. Corrêa AM, da Silva Gonçalves NN, Gonçalves A, Leite GP, Padovani CR. Evolução da relação entre peso e altura e peso e idade em crianças de 3 meses a 6 anos assistidas em creches, Sorocaba (SP), Brasil. Rev Panam Salud Publica 1999;6:26-33.

22. WHO Expert Committee on Physical Status. The use and interpretation of anthropometry: report of a WHO Expert Committee - World Health Organization technical report series 854. Geneva: World Health Organization; 1995.

23. Centers for Disease Control and Prevention [homepage on the internet]. Growth Charts, 2000 [cited 2007 Oct 10]. Available from: http://www.cdc.gov/growthcharts/ 
24. World Health Organization, United Nations Children's Fund, United Nations University. Iron deficiency anaemia: assessment, prevention and control. A guide for programme managers. Geneva: World Health Organization; 2001.

25. De Maeyer EM, Dallman P, Gurney JM, Halberg L, Sood SK, Srikantia SG. Prevenir et combattre l'anémie ferriprive dans le cadre des soins de santé primaires. Génève: World Health Organization; 1991.

26. Bueno MB, Selem SSC, Arêas JAG, Fisberg RM. Prevalência e fatores associado à anemia entre crianças atendidas em creches públicas de São Paulo. Rev Bras Epidemiol 2006;9:462-70.

27. Matta IEA, Veiga GV, Baião MR, Santos MMAS, Luiz RR. Anemia em crianças menores de cinco anos que freqüentam creches públicas do município do Rio de Janeiro, Brasil. Rev Bras Saude Matern Infant 2005;5:349-57.

28. Osório MM, Lira PI, Batista-Filho M, Ashworth A. Prevalence of anemia in children 6-59 months old in the state of Pernambuco, Brazil. Rev Panam Salud Publica 2001;10:101-7.

29. Osório MM. Fatores determinantes da anemia em crianças. J Pediatr (Rio J) 2002;78:269-78

30. Miranda AS, Franceschini SCC, Priore SE, Euclydes MP, Araújo RMA, Ribeiro $\mathrm{SMR}$ et al. Anemia ferropriva e estado nutricional de crianças com idade de 12 a 60 meses do município de Viçosa, MG. Rev Nutr 2003;16:163-9.
31. Fisberg RM, Marchioni DM, Cardoso MR. Estado nutricional e fatores associados ao déficit de crescimento de crianças freqüentadoras de creches públicas do Município de São Paulo, Brasil. Cad Saude Publica 2004;20:812-7.

32. Netto MP. Estado nutricional de ferro e vitaminaAem crianças de 18 a 24 meses do município de Viçosa, Minas Gerais [tese de mestrado]. Viçosa (MG): UFV; 2005.

33. Netto MP, Fonseca MM, Miranda AS, Rocha DS, Silva DG, Franceschini SCC. Relação entre estado nutricional e anemia ferropriva em crianças do município de Viçosa-MG. Rev Pediatr Ceará 2000;1:570.

34. Neuman NA, Tanaka OY, Szarfarc SC, Guimaraes PR, Victora CG Prevalência e fatores de risco para anemia no Sul do Brasil. Rev Saude Publica 2000;34:56-63.

35. Silva DG, Franceschini SCC, Priore SE, Ribeiro SMR, Szarfarc SC, Souza SB et al. Anemia ferropriva em crianças de 6 a 12 meses atendidas na rede pública de saúde do município de Viçosa, Minas Gerais. Rev Nutr 2002;15:301-8.

36. Oliveira RS, Diniz AS, Benigna MJ, Miranda-Silva SM, Lola MM, Gonçalves $\mathrm{MC}$ et al. Magnitude, distribuição espacial e tendência de anemia em préescolares da Paraíba. Rev Saude Publica 2002;36:26-32.

37. Torres MA, Sato K, Queiroz SS. Anemia em crianças menores de dois anos atendidas nas unidades básicas de saúde no Estado de São Paulo, Brasil. Rev Saude Publica 1994;28:290-4. 